\title{
Laparoskopische Appendix-, Hernien- und Kolonchirurgie: State of the Art
}

\author{
R. Bittner, Stuttgart
}

In den 13 Jahren nach der ersten Durchführung der laparoskopischen Cholezystektomie durch Mouret in Lyon ist es zwar weltweit zu einer unvergleichlich stürmischen Verbreitung dieser innovativen chirurgischen Technik gekommen, jedoch sind auch zu Beginn des neuen Jahrtausends noch viele Fragen offen. Wenngleich inzwischen gezeigt werden konnte, daß nahezu alle viszeralchirurgischen Operationen in der neuen Technik möglich sind, so hat sich bisher lediglich die laparoskopische Cholezystektomie als sogenannter Goldstandard durchgesetzt. Allerdings werden aktuell in Deutschland nur 60-70\% aller Gallenblasen laparoskopisch entfernt. Bei der akuten Cholezystitis liegt dieser Prozentsatz noch erheblich niedriger. Darüber hinaus ist eine Konversionsrate von 5 $10 \%$ nicht unüblich. Diese Zahlen signalisieren selbst bei dem als am einfachsten eingeschätzten Verfahren noch erhebliche Ausbildungsdefizite.

Bei der Appendektomie, der Hernienreparation und den kolorektalen Resektionen werden zudem gewichtige grundsätzliche Bedenken angeführt: Bringt die laparoskopische Appendektomie überhaupt Vorteile, da auch konventionell der kleine Schnitt nur $\mathrm{zu}$ einem geringen Bauchdeckentrauma führt? Ist in der Hernienchirurgie der erhöhte technische und kostenintensivere Aufwand gerechtfertigt, wenn diese Operation ebenso in Lokalanästhesie und ambulant durchgeführt werden kann? Kann dem Patienten mit einem kolorektalen Karzinom die laparoskopische Operation zugemutet werden, ohne Kompromisse bezüglich der Radikalität einzugehen? Können Lymphadenektomie und Mesorektumexzision in gleicher Weise durchgeführt werden? Sind Implantationsmetastasen im Bereich der Trokarkanäle ein tatsächliches Problem? Das vorliegende Heft versucht, auf diese wichtigen Fragen eine Antwort zu geben. Einleitend sind die Operationstechniken für Hernienreparation, Appendektomie und Kolonresektion von Arbeitsgruppen aus Kliniken beschrieben, in denen die jeweilige Technik standardisiert und routinemäßig durchgeführt wird. Des weiteren werden die Ergebnisse großer prospektiver klinischer Studien berichtet. Im Mittelpunkt stehen in Anlehnung an die Methode der Metaanalyse die Darstellung und kritische Auswertung der Ergebnisse der bisher publizierten randomisierten Studien. Besondere Beachtung verdient hier die Analyse sämtlicher zur Problematik der Trokarmetastasen publizierten Arbeiten.

Mit diesem Heft, das in seiner Konzeption einzigartig ist, wollen wir einer breiten Leserschaft zum einen Einblick in die bestehende Problematik ausgewählter laparoskopischer Eingriffe im Abdominalbereich geben, zum anderen einen Beitrag zur weiteren Standardisierung und Verbreitung dieser faszinierenden, neuen und innovativen Operationstechnik geben.

\section{KARGER}

Fax +49761 4520714 ๑ 2000 S. Karger GmbH, Freiburg

E-mail Information www.karger.com 